\title{
Set-Systems with Restricted Multiple Intersections
}

\author{
Vince Grolmusz \\ Department of Computer Science \\ Eötvös University, H-1117 Budapest \\ HUNGARY \\ E-mail: grolmusz@cs.elte.hu
}

Submitted: May 30, 2001; Accepted: February 20, 2002.

MR Subject Classifications: 05D05, 05C65, 05D10

\begin{abstract}
We give a generalization for the Deza-Frankl-Singhi Theorem in case of multiple intersections. More exactly, we prove, that if $\mathcal{H}$ is a set-system, which satisfies that for some $k$, the $k$-wise intersections occupy only $\ell$ residue-classes modulo a $p$ prime, while the sizes of the members of $\mathcal{H}$ are not in these residue classes, then the size of $\mathcal{H}$ is at most

$$
(k-1) \sum_{i=0}^{\ell}\left(\begin{array}{l}
n \\
i
\end{array}\right)
$$

This result considerably strengthens an upper bound of Füredi (1983), and gives partial answer to a question of T. Sós (1976).

As an application, we give a direct, explicit construction for coloring the $k$ subsets of an $n$ element set with $t$ colors, such that no monochromatic complete hypergraph on $\exp \left(c(\log m)^{1 / t}(\log \log m)^{1 /(t-1)}\right)$ vertices exists.
\end{abstract}

Keywords: set-systems, algorithmic constructions, explicit Ramsey-graphs, explicit Ramsey-hypergraphs

\section{Introduction}

We are interested in set-systems with restricted intersection-sizes. The famous RayChaudhuri-Wilson [RCW75] and Frankl-Wilson [FW81] theorems give strong upper bounds for the size of set-systems with restricted pairwise intersection sizes. T. Sós asked in 1976 [Sós76], what happens if not the pairwise intersections, but the $k$-wise intersection-sizes are restricted. 
Füredi [Für83], [Für91] showed (actually proving a much more general structure theorem) that for $d$-uniform set-systems over an $n$ element universe, for very small $d$ 's, $(d=O(\log \log n))$, the order of magnitude of the largest set-systems, satisfying $k$-wise or just pairwise intersection restrictions are the same.

In the present paper we strengthen this result of Füredi [Für83]. More exactly, we prove the following $k$-wise version of the Deza-Frankl-Singhi theorem [DFS83]. Note, that no upper bounds for the sizes of sets in the set-system and no uniformity assumptions are made.

Theorem 1 Let $p$ be a prime, let $L \subset\{0,1, \ldots, p-1\}$, and let $k \geq 2$ be an integer. Let $\mathcal{H}$ be a set-system over the $n$ element universe, satisfying that

- (i) $\forall H \in \mathcal{H}: \quad|H| \bmod p \notin L$,

- (ii) $\forall H_{1}, H_{2}, \ldots, H_{k} \in \mathcal{H}$, where $H_{i} \neq H_{j}$ for $i \neq j$ :

$$
\left|H_{1} \cap H_{2} \cap \ldots \cap H_{k}\right| \bmod p \in L,
$$

Then

$$
|\mathcal{H}| \leq(k-1) \sum_{i=0}^{|L|}\left(\begin{array}{c}
n \\
i
\end{array}\right)
$$

As well as in the original Deza-Frankl-Singhi theorem, the upper bound does not depend on $p$, so we can choose a large enough $p$ for proving the non-modular version, $p>n$ certainly suffices.

Our main tool is substituting set-systems into multi-variate polynomials [Gro01]. This tool, together with the linear-algebraic proof of Theorem 9 implies our result.

In the seminal paper of Frankl and Wilson [FW81], the Frankl-Wilson upper bound to the size of a set-system was used for an explicit Ramsey-graph construction. Similarly, we can also use our Theorem 1 to an explicit construction of a $t$-coloring of the edges of the $k$-uniform complete hypergraph, such that no color class will contain a complete, monochromatic hypergraph on a vertex set of size $\exp \left(c(\log n \log \log n)^{1 / t}\right)$. Our explicit construction is similar to the explicit Ramsey-graph construction of [Gro00]. We note, that much better explicit Ramsey hypergraphs can be constructed using the Steppingup Lemma of Erdős and Hajnal [GRS80]: from an explicit construction of $k$-uniform hypergraphs a (much larger) explicit construction of $k+1$-uniform hypergraphs follows, where $k \geq 3$. Another construction for 3-uniform hypergraphs from explicit Ramseygraphs is due to A. Hajnal [Gyá].

Our present Ramsey-hypergraph construction is the best known for 3-uniform hypergraphs with more than 2 colors, and while it is weaker than the (recursive) constructions for $k>3$ with the Stepping-up Lemma of Erdős and Hajnal [GRS80], it is at least direct: does not use constructions for $k$-1-uniform hypergraphs. 


\section{Preliminaries}

Definition 2 ([Gro01]) Let $A=\left\{a_{i j}\right\}$ and $B=\left\{b_{i j}\right\}$ two $u \times v$ matrices over a ring $R$. Their Hadamard-product is an $u \times v$ matrix $C=\left\{c_{i j}\right\}$, denoted by $A \odot B$, and is defined as $c_{i j}=a_{i j} b_{i j}$, for $1 \leq i \leq u, 1 \leq j \leq v$.

Lemma 3 Suppose that $R$ is commutative. Then the Hadamard-product is an associative, commutative and distributive operation:

- $(i)(A \odot B) \odot C=A \odot(B \odot C)$,

- (ii) $A \odot B=B \odot A$,

- $($ iii) $(A+B) \odot C=A \odot C+B \odot C$.

And, for all $\lambda \in R$ :

- $($ iv) $(\lambda A) \odot B=\lambda(A \odot B)$.

We make difference between hypergraphs and set systems over a universe $V$. A hypergraph is a collection of several subsets of $V$, where some subsets may be present with a multiplicity, greater than 1 (called multi-edges). A set system may, however, contain each subset of $V$ at most once.

Definition 4 Let $\mathcal{H}=\left\{H_{1}, H_{2}, \ldots, H_{m}\right\}$ be a hypergraph of $m$ edges (sets) over an $n$ element universe $V=\left\{v_{1}, v_{2}, \ldots, v_{n}\right\}$, and let $U=\left\{u_{i j}\right\}$ be the $n \times m 0$ - 1 incidencematrix of hypergraph $\mathcal{H}$, that is, the columns of $U$ correspond to the sets (edges) of $\mathcal{H}$, the rows of $U$ correspond to the elements of $V$, and $u_{i j}=1$ if and only if $v_{i} \in H_{j}$. The $n \times 1$ incidence-matrix of a single subset $A \subset V$ is called the characteristic vector of $A$.

Note, that every member of a set system is different; so there are no identical columns in an incidence matrix of a set system, but there may be identical columns in an incidence matrix of a hypergraph in case of multi-edges. If $U$ is a $0-1$ matrix with no identical columns, then $U$ is an incidence matrix of a set system.

\subsection{Arithmetic operations on set systems}

Definition 5 Let $f\left(x_{1}, x_{2}, \ldots, x_{n}\right)=\sum_{I \subset\{1,2, \ldots, n\}} a_{I} x_{I}$ be a multi-linear polynomial, where $x_{I}=\prod_{i \in I} x_{i}$. Let $w(f)=\left|\left\{a_{I}: a_{I} \neq 0\right\}\right|$ and let $\mathrm{L}_{1}(f)=\sum_{I \subset\{1,2, \ldots, n\}}\left|a_{I}\right|$.

We need the following definition from [Gro01]: 
Definition 6 ([Gro01]) Let $\mathcal{H}$ be a set-system on the $n$ element universe $V=$ $\left\{v_{1}, v_{2}, \ldots, v_{n}\right\}$ and with $n \times m$ incidence-matrix $U$, and let $f\left(x_{1}, x_{2}, \ldots, x_{n}\right)=$ $\sum_{I \subset\{1,2, \ldots, n\}} a_{I} x_{I}$ be a multi-linear polynomial with non-negative integer coefficients. Then $f\left(\mathcal{H}_{U}\right)$ is a hypergraph on the $\mathrm{L}_{1}(f)$-element vertex-set, and its incidence-matrix is the $\mathrm{L}_{1}(f) \times m$ matrix $W$. The rows of $W$ correspond to $x_{I}$ 's of $f$; there are $a_{I}$ identical rows of $W$, corresponding to the same $x_{I}$. The row, corresponding to $x_{I}$ is defined as the Hadamard-product of those rows of $U$, which correspond to $v_{i}, i \in I$.

Let us remark, that $W$ has rank at most $w(f)$. Also note, that if the coefficients of $x_{1}, x_{2}, \ldots, x_{n}$ are all non-zero, then $f\left(\mathcal{H}_{U}\right)$ is a set-system, since the rows of $U$ is among the rows of the incidence-matrix of $f\left(\mathcal{H}_{U}\right)$.

The crucial property of this operation is given by the following Theorem (Theorem 11 of [Gro01]):

Theorem 7 ([Gro01]) Let $\mathcal{H}=\left\{H_{1}, H_{2}, \ldots, H_{m}\right\}$ be a set-system, and let $U$ be their $n \times m$ incidence-matrix. Let $f$ be a multi-linear polynomial with non-negative integer coefficients, or from coefficients from $\mathbf{Z}_{r}$. Let $f(\mathcal{H})=\left\{\hat{H}_{1}, \hat{H}_{2}, \ldots, \hat{H}_{m}\right\}$. Then, for any $1 \leq k \leq m$ and for any $1 \leq i_{1}<i_{2}<\ldots<i_{k} \leq m$ :

$$
f\left(H_{i_{1}} \cap H_{i_{2}} \cap \ldots \cap H_{i_{k}}\right)=\left|\hat{H}_{i_{1}} \cap \hat{H}_{i_{2}} \cap \ldots \cap \hat{H}_{i_{k}}\right| .
$$

We remark, that in (1) on the left-hand side, $f$ is applied to the characteristic vector (a length- $n$ 0-1 vector) of the set $H_{i_{1}} \cap H_{i_{2}} \cap \ldots \cap H_{i_{k}}$.

\subsection{Multiple intersections}

The proof of the original, pairwise version of the Deza-Frankl-Singhi theorem [DFS83] uses tools from linear algebra: the sets of the set-system $\mathcal{H}$ are associated with independent vectors in a vector space of known dimension; consequently, their number is bounded above by that dimension. Here we also use this idea with some natural modifications.

In the following theorems, the universe of the set-system or the hypergraph is $S=$ $\left\{v_{1}, v_{2}, \ldots, v_{n}\right\}$. When we say hypergraph here, we allow hypergraphs with multi-edges also; consequently, if $F, G$ are two edges of the hypergraph, then we allow that $F$ is the same set, as $G$.

The first step is the following obvious theorem:

Theorem 8 Let $\mathcal{H}=\left\{H_{1}, H_{2}, \ldots, H_{m}\right\}$ be a hypergraph on the $n$-element universe, satisfying $H_{i} \neq \emptyset$ for $i=1,2, \ldots m$. Suppose, that for some positive integer $k \geq 2$, every $k$-wise intersection is empty:

$$
\forall I \subset\{1,2, \ldots, n\},|I|=k: \bigcap_{i \in I} H_{i}=\emptyset
$$

Then

$$
|\mathcal{H}| \leq(k-1) n
$$


Proof: Every element of the universe is in at most $k-1$ sets of $\mathcal{H}$.

We remark, that the above theorem is sharp, as it is shown by $\mathcal{H}=$ $\left\{H_{1}, H_{2}, \ldots, H_{(k-1) n}\right\}$, where $H_{i}=\left\{v_{j}\right\}$, for $i=(j-1)(k-1)+1,(j-1)(k-1)+$ $2, \ldots, j(k-1)$ and $j=1,2, \ldots, n$.

We need the modular version of Theorem 8 . The modular version is an easy exercise for $k=2$; for larger $k$ 's, we need an additional idea.

Theorem 9 Let $p$ be a prime, and let $\mathcal{H}=\left\{H_{1}, H_{2}, \ldots, H_{m}\right\}$ be a hypergraph on the $n$-element universe. Suppose, that $\left|H_{i}\right| \not \equiv 0 \quad(\bmod p)$ for $i=1,2, \ldots, m$, and for some positive integer $k \geq 2$, every $k$-wise intersection-size is zero modulo $p$ :

$$
\forall I \subset\{1,2, \ldots, m\},|I|=k: \bigcap_{i \in I} H_{i} \equiv 0 \quad(\bmod p) .
$$

Then

$$
|\mathcal{H}| \leq(k-1) n_{0} \leq(k-1) n,
$$

if the incidence-vectors of the edges of the hypergraph $\mathcal{H}$ span an $n_{0} \leq n$-dimensional subspace of the $n$-dimensional vector-space over $G F(p)$.

Proof: $\quad$ For $i=1$ through $m$, let $x^{(i)} \in\{0,1\}^{n}$ denote the characteristic vector of set $H_{i}$. In the case of $k=2$, it is easy to see that their dot-product, $x^{(i)} \cdot x^{(j)}$, is zero modulo $p$ if $i \neq j$, and non-zero otherwise; thus vectors $x^{(i)}, i=1,2, \ldots, m$ are independent in an $n_{0}$-dimensional subspace, so $m \leq n_{0}$.

We generalize this proof for larger values of $k$. Obviously, $\left|H_{i} \cap H_{j}\right|=x^{(i)} \cdot x^{(j)}$. This can also be written as $\left|H_{i} \cap H_{j}\right|=\left(x^{(i)} \odot x^{(j)}\right) \cdot \mathbf{1}$, where $\mathbf{1}$ denotes the length- $n$ all-1 vector, and $x^{(i)} \odot x^{(j)}$ is the characteristic vector of $H_{i} \cap H_{j}$. Now it is easy to see, that the characteristic vector of

$$
\bigcap_{i \in I} H_{i}
$$

is

$$
\bigodot_{i \in I} x^{(i)}
$$

consequently,

$$
\left|\bigcap_{i \in I} H_{i}\right|=\bigodot_{i \in I} x^{(i)} \cdot \mathbf{1}
$$

Let $z^{(i)}$, for $i=1,2, \ldots, k, n$-dimensional vectors. Let us define

$$
g\left(z^{(1)}, z^{(2)}, \ldots, z^{(k)}\right)=\left(\bigodot_{i=1}^{k} z^{(i)}\right) \cdot \mathbf{1} .
$$

In particular,

$$
g\left(x^{\left(i_{1}\right)}, x^{\left(i_{2}\right)}, \ldots, x^{\left(i_{k}\right)}\right)=\left|\bigcap_{j=1}^{k} H_{i_{j}}\right| .
$$


Consequently, from our assumptions, if $i_{s} \neq i_{t}$ for $s \neq t$, then

$$
g\left(x^{\left(i_{1}\right)}, x^{\left(i_{2}\right)}, \ldots, x^{\left(i_{k}\right)}\right) \equiv 0 \quad(\bmod p)
$$

while for all $i=1,2, \ldots, m$ :

$$
g\left(x^{(i)}, x^{(i)}, \ldots, x^{(i)}\right) \not \equiv 0 \quad(\bmod p) .
$$

From Lemma 3, $g$ is a multi-linear function. We need the following Lemma to conclude the proof:

Lemma 10 Let $U \subset V$, where $V$ is a vector-space over the field $F$. Suppose, that vectors in $U$ generates an $n_{0}$-dimensional subspace of $V$, also assume that $|U| \geq n_{0}(k-1)+$ 1. Then there exists an $u \in U$, such that $u$ can be written $k$ different ways as the linear combinations of vectors from $U$ such that no vector appears in two of these linear combinations.

In other words, the Lemma states that there exist pairwise disjoint subsets $W_{1}, W_{2}, \ldots, W_{k} \subset U$, such that

$$
u=\sum_{v \in W_{1}} a_{v} v=\sum_{v \in W_{2}} a_{v} v=\cdots=\sum_{v \in W_{k}} a_{v} v
$$

for $a_{v} \in F$.

Proof: Let $W_{1}$ be a maximal linear independent vector-set from $U$, and for $j=$ $2,3, \ldots, k-1$, let $W_{j}$ be a maximal linear independent vector-set from $U-\left(W_{1} \cup W_{2} \cup\right.$ $\left.\ldots \cup W_{j-1}\right)$. Since $\left|W_{i}\right| \leq n_{0}$ for $i=1,2, \ldots, k-1$, there exists a $u$ such that $u \in$ $U-\left(W_{1} \cup W_{2} \cup \ldots \cup W_{k-1}\right)$. Let us define $W_{k}=\{u\}$.

Now, for $i=1,2, \ldots, k-1$, set $W_{i} \cup\{u\}$ is dependent, while $W_{i}$ is not, and we are done.

Now we give an indirect proof for the theorem. Suppose, that $|\mathcal{H}| \geq(k-1) n_{0}+1$. Apply Lemma 10 to $U=\left\{x^{(1)}, x^{(2)}, \ldots, x^{\left((k-1) n_{0}+1\right)}\right\}$. Now, there exists a $u \in U$, such that $u$ can be given as $k$ linear combinations of disjoint vector-subsets of $U$. Since $u=x^{(i)}$, for some $i$, from $(5)$,

$$
g(u, u, \ldots, u) \not \equiv 0 \quad(\bmod p) .
$$

But, on the other hand, $u$ can be given in $k$ linear combinations, each containing vectors from pairwise disjoint vector sets. Consequently, by the multi-linearity of $g, g(u, u, \ldots, u) \not \equiv 0(\bmod p)$ can be written as a linear combination of numbers $g\left(x^{\left(i_{1}\right)}, x^{\left(i_{2}\right)}, \ldots, x^{\left(i_{k}\right)}\right)$, where $i_{s} \neq i_{t}$ for $s \neq t$. By $(4)$, all of these numbers are 0 modulo $p$, so their linear combination is also zero modulo $p$, and this contradicts to (6).

\subsection{Proof of the main theorem}

Now we have all the tools needed for the proof of Theorem 1. Certainly, $L \neq \emptyset$. Let

$$
g(x)=\prod_{a \in L}(x-a) .
$$

THE ELECTRONic JOURNAL OF COMBinatorics 9 (2002), \#R8 
Now let $f$ be the unique multi-linear polynomial over $\operatorname{GF}(p)$, such that

$$
f\left(x_{1}, x_{2}, \ldots, x_{n}\right)=g\left(x_{1}+x_{2}+\cdots+x_{n}\right) .
$$

The degree of $f$ is at most $|L|$, so $\mathrm{L}_{1}(f) \leq(p-1) \sum_{i=0}^{|L|}\left(\begin{array}{c}n \\ i\end{array}\right)$, and $w(f) \leq \sum_{i=0}^{|L|}\left(\begin{array}{c}n \\ i\end{array}\right)$. Consider now hypergraph $f(\mathcal{H})$. The vertex-set of this hypergraph is of size $\mathrm{L}_{1}(f)$, and the incidence-vectors of the edges span a $w(f)$-dimensional subspace $U$ of the $\mathrm{L}_{1}(f)$ dimensional vector space $V$. By Theorem 7, hypergraph $f(\mathcal{H})$ satisfies the assumptions of Theorem 9, so

$$
|\mathcal{H}|=|f(\mathcal{H})| \leq(k-1)\left(\sum_{i=0}^{|L|}\left(\begin{array}{l}
n \\
i
\end{array}\right)\right)
$$

\section{Set-systems with restricted $k$-wise intersections}

In this section we give an explicit construction for a set-system with similar (but stronger) properties described in [Gro00].

It was conjectured (see [BF92]), that if $\mathcal{H}$ is a set-system over an $n$ element universe, satisfying that $\forall H \in \mathcal{H}:|H| \equiv 0 \quad(\bmod 6)$, but $\forall G, H \in \mathcal{H}, G \neq H:|G \cap H| \not \equiv 0$ (mod 6) has size polynomial in $n$. The conjecture was motivated by theorems of Frankl and Wilson, showing polynomial upper bounds for prime or prime-power moduli [FW81]. We have shown in [Gro00] that there exists an $\mathcal{H}$ with these properties and with superpolynomial size in $n$. (see the details in [Gro00].) In [Gro01] we gave this construction with the notions of Definition 6 . Here we present a $k$-wise intersection-version, which will be useful for a Ramsey hypergraph construction. On the other hand, this construction will also show, that our Theorem 1 does not generalize to non-prime-power composite moduli.

Theorem 11 Let $n, t \geq 2$ integers, and let $p_{1}, p_{2}, \ldots, p_{t}$ be pairwise different primes, and let $q=p_{1} p_{2} \cdots p_{t}$. There exists an explicitly constructible set-system $\mathcal{H}=$ $\left\{H_{1}, H_{2}, \ldots, H_{m}\right\}$ on the n-element universe, such that

(i) $|\mathcal{H}|=m \geq \exp \left(\frac{c(\log n)^{t}}{(\log \log n)^{t-1}}\right)$

(ii) $\forall H \in \mathcal{H},|H| \equiv 0 \quad(\bmod q)$,

(iii) $\forall I \subset\{1,2, \ldots, m\}, 2 \leq|I|,\left|\bigcap_{i \in I} H_{i}\right| \not \equiv 0 \quad(\bmod q)$.

\section{Proof:}

Let $s$ be a positive integer, and for $i=1,2, \ldots, t$ let $\alpha_{i}$ be the smallest integer that $s<p_{i}^{\alpha_{i}}$. By a result of Barrington, Beigel and Rudich [BBR94], for any $\ell \geq s$ there 
exists an explicitly constructible $\ell$-variable, degree- $O(s)$ polynomial $f$, satisfying over $x=\left(x_{1}, x_{2}, \ldots, x_{\ell}\right) \in\{0,1\}^{\ell}:$

$$
f(x) \equiv 0 \quad(\bmod q) \Longleftrightarrow \sum_{i=1}^{\ell} x_{i} \equiv 0 \quad\left(\bmod p_{1}^{\alpha_{1}} p_{2}^{\alpha_{2}} \cdots p_{t}^{\alpha_{t}}\right) .
$$

Let $r=p_{1}^{\alpha_{1}} p_{2}^{\alpha_{2}} \cdots p_{t}^{\alpha_{t}}$, and let $\mathcal{G}_{0}$ denote the set-system of all $r-1$-element subsets of the $\ell-1$-element universe. Let us take an additional element $e$ outside this universe, and let us define set-system $\mathcal{G}=\left\{G \cup\{e\} G \in \mathcal{G}_{0}\right\}$. Indeed, for any $k \geq 2$, all $k$-wise intersections in $\mathcal{G}$ are non-empty, and of size less than $r$, while the size of any element of $\mathcal{G}$ is exactly $r$.

Then consider $\mathcal{H}=f(\mathcal{G})$. By Theorem $7, \mathcal{H}$ satisfies (ii) and (iii), and since the $f$ of Barrington, Beigel and Rudich [BBR94] contains all variable $x_{i}$ with a non-zero coefficient, then $\mathcal{H}$ is a set-system. The size of $\mathcal{H}$ is the same as the size of $\mathcal{G}$ :

$$
\left(\begin{array}{l}
\ell-1 \\
r-1
\end{array}\right)
$$

Now set $\ell=r^{2}$, then

$$
|\mathcal{H}|=|\mathcal{G}|=\left(\begin{array}{c}
r^{2} \\
r-1
\end{array}\right) \geq r^{r}
$$

The size of the universe of $\mathcal{H}=f(\mathcal{G})$ is

$$
n=\mathrm{L}_{1}(f)=\ell^{O(s)}=r^{O\left(r^{1 / t}\right)},
$$

so

$$
|\mathcal{H}|=\exp \left(\frac{c(\log n)^{t}}{(\log \log n)^{t-1}}\right),
$$

for some positive constant $c$, depending only on $q$ (or the primes $p_{1}, p_{2}, \ldots, p_{t}$ ).

\section{An Explicit Ramsey-Hypergraph Construction}

Theorem 12 Let $m, k, t \geq 2$ integers. Let $\mathcal{F}$ denote the complete $k$-uniform set-system on the m-element universe $S$. Then there exists an explicitly constructible t-coloring of the sets of the $k$-uniform set-system $\mathcal{F}$ which does not contain monochromatic complete sub-system on

$$
\exp \left(c(\log m)^{1 / t}(\log \log m)^{1 /(t-1)}\right)
$$

vertices. 
Proof: First construct a set-system $\mathcal{H}$ with Theorem 11 with the first $t$ primes: $p_{1}=$ $2, p_{2}=3, \ldots, p_{t}$. Set $S=\mathcal{H}$. (If $m$ is not exactly the size of $\mathcal{H}$, then generate the smallest $\mathcal{H}$ with at least $m$ elements, and let $S \subset \mathcal{H}$.) Consequently, a member of our set-system $F \in \mathcal{F}$ corresponds to $k$ sets of $\mathcal{H}: F=\left\{H_{1}, H_{2}, \ldots, H_{k}\right\}$.

Next we define the coloring of $\mathcal{F}$.

Color $F$ to color $c_{v},(1 \leq v \leq t)$ if $v$ is the smallest number that $p_{v}$ does not divide

$$
\left|\bigcap_{i=1}^{k} H_{i}\right| \text {. }
$$

Clearly, every $F$ will have some color. If every $k$-set in $S^{\prime} \subset S$ is of color $c_{v}$, then apply Theorem 1 with $p=p_{v}$, and get the upper bound.

\section{Acknowledgment.}

The author is indebted to Zoltán Füredi, András Gyárfás and Lajos Rónyai for discussions on this topic. Part of this research was done while visiting the DIMACS Center in Piscataway, NJ. The author also acknowledges the partial support of Janos Bolyai Fellowship, of Farkas Bolyai Fellowship, and research grants FKFP 0607/1999, and OTKA T030059.

\section{References}

[BBR94] David A. Mix Barrington, Richard Beigel, and Steven Rudich. Representing Boolean functions as polynomials modulo composite numbers. Comput. Complexity, 4:367-382, 1994. Appeared also in Proc. 24th Ann. ACM Symp. Theor. Comput., 1992.

[BF92] László Babai and Péter Frankl. Linear algebra methods in combinatorics. Department of Computer Science, The University of Chicago, September 1992. preliminary version.

[DFS83] M. Deza, P. Frankl, and N. M. Singhi. On functions of strength $t$. Combinatorica, 3:331-339, 1983.

[Für83] Z. Füredi. On finite set-systems whose every intersection is a kernel of a star. Discrete Math., 47(1):129-132, 1983.

[Für91] Zoltán Füredi. Turán type problems. In Surveys in combinatorics, 1991 (Guildford, 1991), pages 253-300. Cambridge Univ. Press, Cambridge, 1991.

[FW81] Péter Frankl and R. M. Wilson. Intersection theorems with geometric consequences. Combinatorica, 1(4):357-368, 1981.

[Gro00] Vince Grolmusz. Superpolynomial size set-systems with restricted intersections mod 6 and explicit Ramsey graphs. Combinatorica, 20:73-88, 2000. 
[Gro01] Vince Grolmusz. Constructing set-systems with prescribed intersection sizes. Technical Report DIMACS TR 2001-03, DIMACS, January 2001. ftp://dimacs.rutgers.edu/pub/dimacs/TechnicalReports/TechReports/2001/200103.ps.gz.

[GRS80] Ronald L. Graham, Bruce L. Rothschild, and Joel H. Spencer. Ramsey Theory. John Wiley \& Sons, 1980.

[Gyá] András Gyárfás. personal communication.

[RCW75] D. K. Ray-Chaudhuri and R. M. Wilson. On t-designs. Osaka J. Math., 12:735$744,1975$.

[Sós76] Vera T. Sós. Some remarks on the connection of graph theory, finite geometry and block designs. In Teorie Combinatorie; Proc. of the Colloq. held in Rome 1973, pages 223-233, 1976. 